\title{
Efeito da densidade de estocagem do biofiltro na capacidade de filtração de
}

\section{nutrientes da Ulva lactuca}

\author{
Effect of biofilter storage density on the nutrient filtration capacity of Ulva lactuca
}

Efecto de la densidad de almacenamiento del biofiltro en la capacidad de filtración de nutrientes de

\section{Ulva lactuca}

Recebido: 26/01/2022 | Revisado: 03/02/2022 | Aceito: 10/02/2022 | Publicado: 16/02/2022

\author{
Ricardo de Oliveira Soares \\ ORCID: https://orcid.org/0000-0001-8635-4982 \\ Fundação Instituto de Pesca do Estado do Rio de Janeiro, Brasil \\ E-mail: ricardoos.89@gmail.com \\ Beatriz Castelar \\ ORCID: https://orcid.org/0000-0002-0025-556X \\ d'Alga aquicultura urbana, Brasil \\ E-mail: beatrixcastelar@gmail.com \\ Marcelo Duarte Pontes \\ ORCID: https://orcid.org/0000-0003-3344-1411 \\ Fundação Instituto de Pesca do Estado do Rio de Janeiro, Brasil \\ E-mail: marcelodpontes@gmail.com
}

\section{Resumo}

O presente trabalho buscou avaliar a capacidade de filtração de nitrogênio e fósforo inorgânicos por biofiltro da macroalga Ulva lactuca em função da densidade de estocagem em sistema de AMTI em recirculação de água. As macroalgas foram cultivadas em 20 unidades experimentais, com 5 densidades de estocagem $\left(2,4,6,8\right.$ e $\left.10 \mathrm{~kg} . \mathrm{m}^{-3}\right) \mathrm{e}$ 4 repetições. As concentrações dos nutrientes amônia $\left(\mathrm{N}-\mathrm{NH}_{3}\right)$, nitrito $\left(\mathrm{N}-\mathrm{NO}_{2}\right)$, nitrato $\left(\mathrm{N}^{\left.-\mathrm{NO}_{3}\right)}\right.$ e fosfato $\left(\mathrm{P}-\mathrm{PO}_{4}\right)$ foram analisadas no momento da interrupção do abastecimento (06:00) e após 12 horas (18:00), para avaliação da filtração de nutrientes. Não foram observadas variações significativas $(p>0,05)$ entre os valores da eficiência de filtração de nitrogênio (EF-N) e fósforo (EF-P) e da taxa de filtração de nitrogênio (TF-N) e fósforo (TF-P) em função da densidade de estocagem. Porém, foi observado efeito $(p<0,05)$ da densidade de estocagem sobre o índice de filtração de nitrogênio (IF-N) e fósforo (IF-P), onde os maiores índices foram observados para as menores densidades de estocagem. A densidade de estocagem da macroalga interfere na capacidade de filtração de nutrientes, onde as menores densidades de estocagem proporcionam maior capacidade de filtração da macroalga.

Palavras-chave: Sistema de Aquicultura Multitrófica Integrada; Macroalga; Agricultura marinha; Aquicultura sustentável.

\begin{abstract}
This work aimed to evaluate the filtering capacity of inorganic nitrogen and phosphorus by biofilter of the macroalgae Ulva lactuca as a function of stocking density in an AMTI system in water recirculation. The macroalgae were cultivated in 20 experimental units, with 5 stocking densities $\left(2,4,6,8\right.$ and $\left.10 \mathrm{~kg} . \mathrm{m}^{-3}\right)$ and 4 replications. The concentrations of the nutrients ammonia $\left(\mathrm{N}-\mathrm{NH}_{3}\right)$, nitrite $\left(\mathrm{N}-\mathrm{NO}_{2}\right)$, nitrate $\left(\mathrm{N}-\mathrm{NO}_{3}\right)$ and phosphate $\left(\mathrm{P}-\mathrm{PO}_{4}\right)$ were analyzed at the time of interruption of supply (06:00) and after 12 hours (18:00) for evaluation of nutrient filtration. There were no significant variations $(\mathrm{p}>0.05)$ between the values of filtration efficiency of nitrogen (EF-N) and phosphorus (EF-P) and of the filtration rate of nitrogen (TF-N) and phosphorus (TF- P) as a function of stocking density. However, an effect ( $\mathrm{p}<0.05)$ of stocking density on the filtration index of nitrogen (IF-N) and phosphorus (IFP) was observed, where the highest indexes were observed for the lowest stocking densities. The macroalgae storage density interferes with the nutrient filtration capacity, where lower storage densities provide greater macroalgae filtration capacity.
\end{abstract}

Keywords: Integrated Multitrophic Aquaculture System; Macroalgae; Marine agriculture; Sustainable aquaculture

\section{Resumen}

Este trabajo tuvo como objetivo evaluar la capacidad de filtrado de nitrógeno y fósforo inorgánicos por biofiltro de la macroalga Ulva lactuca en función de la densidad de población en un sistema AMTI en recirculación de agua. Las macroalgas se cultivaron en 20 unidades experimentales, con 5 densidades de almacenamiento $\left(2,4,6,8\right.$ y $\left.10 \mathrm{~kg} \cdot \mathrm{m}^{-3}\right)$ y 4 repeticiones. Las concentraciones de los nutrientes amonio $\left(\mathrm{N}-\mathrm{NH}_{3}\right)$, nitrito $\left(\mathrm{N}-\mathrm{NO}_{2}\right)$, nitrato $\left(\mathrm{N}^{-} \mathrm{NO}_{3}\right)$ y fosfato $\left(\mathrm{P}^{-} \mathrm{PO}_{4}\right)$ se analizaron en el momento de la interrupción del suministro (06:00) y después de las 12 horas (18:00) para la evaluación de la filtración de nutrientes. No hubo variaciones significativas ( $>0.05)$ entre los valores de eficiencia 
de filtración de nitrógeno (EF-N) y fósforo (EF-P) y de la tasa de filtración de nitrógeno (TF-N) y fósforo (TF-P ) en función de la densidad de población. Sin embargo, se observó un efecto $(\mathrm{p}<0.05)$ de la densidad de población sobre el índice de filtración de nitrógeno (IF-N) y fósforo (IF-P), donde se observaron los índices más altos para las densidades de carga más bajas. La densidad de almacenamiento de las macroalgas interfiere con la capacidad de filtración de nutrientes, donde las densidades de almacenamiento más bajas proporcionan una mayor capacidad de filtración de las macroalgas.

Palabras clave: Sistema Integrado de Acuicultura Multitrófica; Macroalgas; Agricultura marina; Acuicultura sostenible.

\section{Introdução}

O desenvolvimento de novas tecnologias na aquicultura tem possibilitado o crescimento constante da produção de peixes nas últimas décadas, fazendo desta atividade uma das mais importantes para a segurança alimentar mundial (FAO, 2020). Entretanto, a intensificação dos sistemas de produção, com densidades de estocagem cada vez mais elevadas, têm contribuído para o aumento da eutrofização dos ambientes naturais aquáticos (Boyd, 2003).

Outro fator importante para o aumento da eutrofização causada pela aquicultura é a qualidade do efluente, em geral enriquecido em nitrogênio. Isso porque peixes retém em sua carcaça uma pequena fração dos nutrientes fornecidos na ração. Em geral, os peixes necessitam ingerir $458 \mathrm{~g}$ de proteína para cada $\mathrm{kg}$ de biomassa produzida e a retenção da proteína na carcaça é de apenas 35\% (Oliva-Teles, et al., 2020). Em acompanhamento de um ciclo de produção de Tilápia (Oreochromis niloticus) observou-se que somente $30 \%$ do nitrogênio e $32 \%$ do fósforo fornecidos na alimentação foram convertidos em biomassa pelo peixe (Boyd \& Queiroz, 2004).

Embora a maior parte das aquiculturas de peixes ainda utilize tanques escavados ou tanques redes como sistema de produção, cresce o interesse pela utilização de sistemas mais sustentáveis, com reaproveitamento da água, tais como sistemas de recirculação (RAS) e a aquaponia, no qual os nutrientes perdidos pelos peixes são reaproveitados para a produção de vegetais (Goodek, et al., 2018). A aquaponia, assim como outros sistemas de aquicultura multitrófica integrada (AMTI), associa em um mesmo sistema de cultivo espécies de diferentes níveis tróficos com interesse comercial, de modo que os nutrientes não aproveitados em cada cultivo são aproveitados por outro nivel trófico, diversificando assim, os produtos rentáveis daquele sistema de produção.

Nos sistemas de AMTI marinhos as macroalgas e as plantas halófitas são os organismos mais adequados para exercer a função de biofiltro. No entanto, para a maioria das AMTI, as tecnologias de produção ainda estão em fase de desenvolvimento. No caso das macroalgas, a combinação de diversos fatores abióticos, como a intensidade luminosa, a salinidade, a temperatura e a disponibilidade de nutrientes determina o seu desenvolvimento e composição química (Chopin, et al., 2001; Hayashi, et al., 2008; He, et al., 2020). Além disso, a taxa de renovação da água, o sistema de aeração e a densidade de estocagem são parâmetros de cultivo que podem influenciar o crescimento da macroalga (Msuya \& Neori, 2008; Al-Hafedh, et al., 2014; Ben-Ari, et al., 2014) e até seu valor nutricional (Queirós, et al., 2021), com grande influência no seu desempenho como biofiltro. Deste modo, o presente trabalho buscou estabelecer uma densidade de estocagem que maximize o desempenho de um biofiltro de macroalga Ulva lactuca em sistema de RAS.

\section{Metodologia}

O ensaio de capacidade de retenção de nutrientes foi realizado nas dependências da empresa d’Alga Aquicultura Urbana, localizada na cidade do Rio de Janeiro - RJ. O sistema de cultivo possui $40 \mathrm{~m}^{3}$ de volume de água, composto por um tanque circular lona PVC (Policloreto de Vinila) e estrutura de arame galvanizado, com 6,4 m de diâmetro e $27 \mathrm{~m}^{3}$ de capacidade. Neste tanque foi cultivado tilápia (Oreochromis niloticus) com uma biomassa total estimada de $20 \mathrm{~kg}$. Os peixes foram alimentados diariamente com ração de $32 \%$ de proteína bruta (PB). A água do fundo desse tanque é drenada por 
gravidade para um tanque de decantação de $2 \mathrm{~m}^{3}$, onde foi bombeada (bomba 1/4cv, SYLLENT) para o sistema de filtros UVc (4*30 W, CLEANJUMP) e então distribuída por derivação para a bateria de tanques de cultivo de macroalgas. Os referidos tanques (UE) tem abastecimento individual com vazão de 1,33 L. $\mathrm{min}^{-1}$, regulada com o auxílio de gotejadores. Possuem sistema de aeração formado por um soprador de ar central $\left(54 \mathrm{~m}^{3} \cdot \mathrm{H}^{-1}\right.$, RESUN), e distribuído para as UE com mangueiras microperfuradas $(60 \mathrm{~cm})$. Os tanques são de polipropileno cor azul, com $0,08 \mathrm{~m}^{3}$ de capacidade e $0,45 \mathrm{~m}^{2}$ de superfície. O efluente dos tanques de cultivo de macroalgas é drenado por gravidade e retornam para os tanques de cultivo de peixes.

A temperatura e a irradiância foram monitoradas a cada hora com auxílio de data loggers (HOBO Pendant, UA-00208). As macroalgas foram cultivadas em $20 \mathrm{UE}$, com 5 densidades de estocagem $\left(2,4,6,8\right.$ e $\left.10 \mathrm{~kg} \cdot \mathrm{m}^{-3}\right)$ e 4 repetições distribuídas aleatoriamente. A salinidade foi medida com o auxílio de um refratômetro manual (VODEX, VX100SG) na hora da primeira coleta (06:00).

As concentrações de nitrogênio inorgânico dissolvido (NID) representado pelo somatório das concentrações de amônia (N-NH$)$, nitrito $\left(\mathrm{N}-\mathrm{NO}_{2}\right)$ e nitrato $\left(\mathrm{N}^{\left.-\mathrm{NO}_{3}\right)}\right.$ além da concentração de fósforo inorgânico dissolvido (PID), representado pela concentração de fosfato $\left(\mathrm{P}^{-} \mathrm{PO}_{4}\right)$ foram analisadas por método colorimétrico com auxílio de um fotômetro (HANNA, HI 83203). Para a avaliação, as UE tiveram o abastecimento de água interrompido, permanecendo em sistema estático durante todo o período de avaliação da retirada de nutrientes. A avaliação da filtração de nutrientes da água teve início às 06:00hs do dia seguinte aos manejos de pesagem e correção da densidade de estocagem em cada UE. Foi avaliada a concentração de nutrientes inicial, com a coleta de quatro amostras de água no momento da interrupção do abastecimento e após 12 horas de experimento (18:00) uma amostra da água de cada UE foi coletada para avaliação da retirada no período diurno.

o desempenho de biofiltração das algas foi estimado através da eficiência de filtração de nutrientes (EF), taxa de filtração de nutrientes (TF) e índice de filtração (IF) através das fórmulas:

\section{$E F(\%)=([$ nutriente inicial] $-[$ nutriente final $]) /[$ nutriente inicial] $\times 100$ \\ $T F\left(m g \cdot L^{-1} \cdot h^{-1}\right)=([$ nutriente inicial] - [nutriente final $]) /$ tempo \\ IF $\left(m g . h^{-1}\right)=(T F /$ densidade de estocagem $)$}

Diferenças em EF, TF e IF, entre os tratamentos foram testadas através de análise de variâncias (ANOVA) e as significâncias identificadas através do teste de Tukey, com auxílio do software Statistica 7.0. Os dados foram apresentados em média \pm desvio padrão e o nível de tolerância para os testes de significância foi 0,05 .

\section{Resultados}

Os valores médios (mg. $\mathrm{L}^{-1} \pm$ desvio padrão) iniciais dos nutrientes no AMTI foram de $0,35 \pm 0,06 \mathrm{~N}^{-\mathrm{NH}_{3}} ; 9,10 \pm 0,91$ $\mathrm{N}-\mathrm{NO}_{2} ; 267,60 \pm 35,63 \mathrm{~N}-\mathrm{NO}_{3}$ e 4,18 \pm 0,84 de P - $\mathrm{PO}_{4}$, ou seja, 277,05 mg.L.-1 de NID e 4,18 mg.L-1 de PID. Os valores finais de NID e PID, bem como da eficiência de filtração, taxa de filtração e do índice de filtração de nitrogênio e fósforo para cada uma das densidades de estocagem testadas estão apresentados na tabela 1. Não foram observadas diferenças significativas ( $p>0,05)$ entre os valores da EF e da TF de nutrientes em função da densidade de estocagem. Porém, foi observado efeito $(\mathrm{p}<0,05)$ da densidade de estocagem sobre o índice de filtração (IF), onde os maiores índices foram observados para as menores densidades de estocagem. 
Tabela 1: Valores médios ( \pm desvio padrão) das concentrações de nitrogênio inorgânico dissolvido final (NID final), fósforo inorgânico dissolvido final (PID final), da eficiência de filtração de nitrogênio (EF-N) e fósforo (EF-P), taxa de filtração de nitrogênio (TF-N) e fósforo (TF-P) e índice de filtração de nitrogênio (IF-N) e fósforo (IF-P) em função da densidade de estocagem da macroalga Ulva lactuca em biofiltros em sistema de aquicultura multitrófica integrada em recirculação de água.

\begin{tabular}{|c|c|c|c|c|c|}
\hline & \multicolumn{5}{|c|}{ Densidade de estocagem da Ulva lactuca $\left(\mathrm{kg} \cdot \mathrm{m}^{-3}\right)$} \\
\hline & 2 & 4 & 6 & 8 & 10 \\
\hline NID final (mg.L $\left.\mathrm{L}^{-1}\right)$ & $130,91 \pm 64,66$ & $102,95 \pm 49,62$ & $74,50 \pm 38,17$ & $96,76 \pm 34,80$ & $94,84 \pm 45,01$ \\
\hline PID final (mg. $\left.\mathrm{L}^{-1}\right)$ & $0,93 \pm 0,39$ & $0,58 \pm 0,15$ & $1,03 \pm 0,19$ & $0,80 \pm 0,22$ & $0,85 \pm 0,30$ \\
\hline EF-N $(\%)$ & $54,14 \pm 24,71$ & $64,02 \pm 17,25$ & $69,88 \pm 10,51$ & $65,63 \pm 12,40$ & $66,97 \pm 16,47$ \\
\hline TF-N (mg. $\left.\mathrm{L}^{-1} \cdot \mathrm{h}^{-1}\right)$ & $13,40 \pm 6,80$ & $15,27 \pm 4,13$ & $13,43 \pm 3,13$ & $15,44 \pm 3,09$ & $16,24 \pm 4,35$ \\
\hline IF-N (mg.h $\left.\mathrm{h}^{-1}\right)$ & $6,70 \pm 3,40 \mathrm{a}$ & $3,82 \pm 1,03 \mathrm{ab}$ & $2,24 \pm 0,52 b$ & $1,93 \pm 0,39 b$ & $1,62 \pm 0,43 b$ \\
\hline $\mathrm{EF}-\mathrm{P}(\%)$ & $77,46 \pm 13,92$ & $84,40 \pm 5,15$ & $72,02 \pm 12,54$ & $78,81 \pm 7,26$ & $82,02 \pm 8,53$ \\
\hline TF-P (mg.L $\left.L^{-1} \cdot h^{-1}\right)$ & $0,30 \pm 0,12$ & $0,27 \pm 0,04$ & $0,23 \pm 0,06$ & $0,26 \pm 0,07$ & $0,34 \pm 0,08$ \\
\hline IF-P $\left(m g \cdot h^{-1}\right)$ & $0,15 \pm 0,06 \mathrm{a}$ & $0,07 \pm 0,01 b$ & $0,04 \pm 0,01 b$ & $0,03 \pm 0,01 b$ & $0,03 \pm 0,01 b$ \\
\hline
\end{tabular}

Fonte: Autores (2022).

Durante o período experimental, a salinidade da água nas UEs foi de $30 \pm 0,2 \% \mathrm{ppm}$. A temperatura variou de 21,2 a $34,2^{\circ} \mathrm{C}$ e irradiância média foi $300 \mu \mathrm{mol}$ fótons $\cdot \mathrm{m}^{-2}$. $\mathrm{s}^{-1}$, com pico de $680,6 \mu \mathrm{mol}$ fótons. $\mathrm{m}^{-2} \cdot \mathrm{s}^{-1}$ às $11 \mathrm{~h}$, conforme representado na Figura 1.

Figura 1: Variação da temperatura $\left({ }^{\circ} \mathrm{C}\right)$ da água e irradiância $\left(\mu \mathrm{mol}\right.$ de fótons $\left.\cdot \mathrm{m}^{-2} \cdot \mathrm{s}^{-1}\right)$ na superfície dos tanques de cultivo, durante o período experimental.

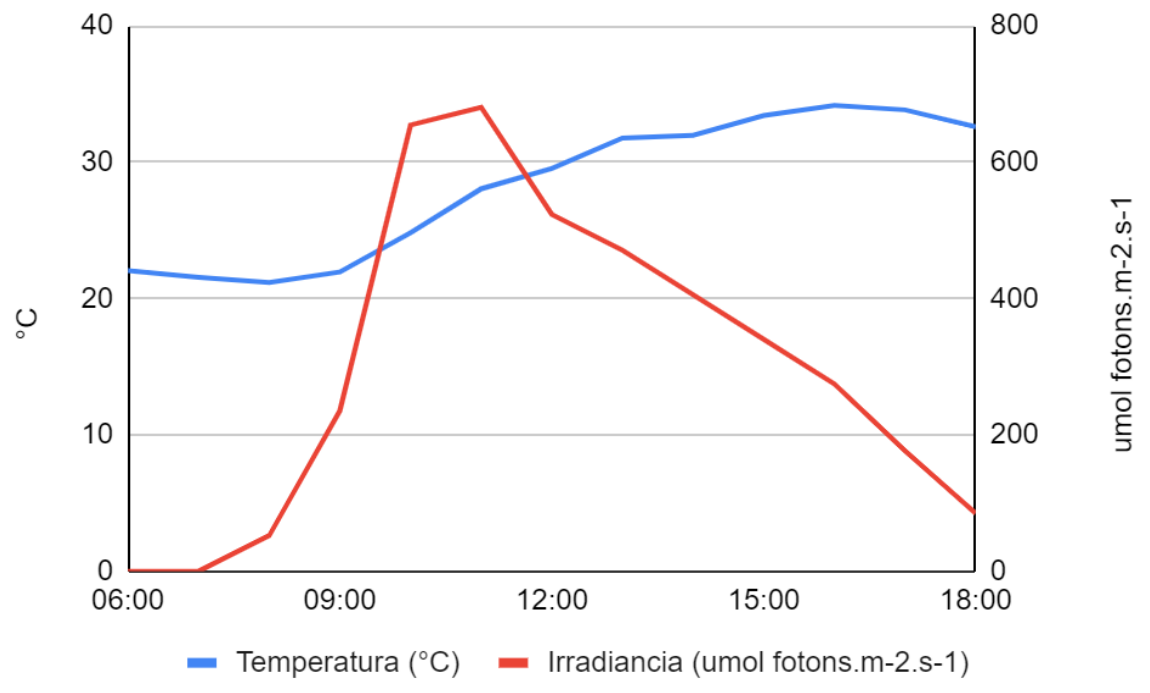

Fonte: Autores (2022).

\section{Discussão}

A macroalga $U$. lactuca apresentou bom desempenho de filtração de nitrogênio e fósforo em IMTA com tilápia, independentemente da densidade de estocagem. Cerca de $64 \%$ de todo o NID e $78 \%$ de todo o PID disponível inicialmente foram filtrados durante as 12 horas experimentais. Foram absorvidos, em média, 14,75 mg de NID e 0,28 mg de PID por hora em cada litro do sistema de biofiltros.

Estes valores evidenciam o potencial da Ulva na fitorremediação dos efluentes da aquicultura. Em um sistema de 
tanques sequenciais de Ulva fasciata $88 \%$ de todo o NID do efluente oriundo do tanque de cultivo da tainha (Mugil cephalus) (Shahar \& Guttman, 2021). A Ulva apresentou maior capacidade de retirada de nitrogenados de efluentes de piscicultura marinha em comparação com Sargassum sp., Gelidium sp., e Dictyota sp. (Adharini, et al., 2021).

A Figura 2 representa a capacidade de filtração das excretas solúveis de uma produção hipotética de tilápia, na fase de terminação, alimentadas diariamente com $1 \mathrm{~kg}$ de ração. Aproximadamente um metro cúbico de biofiltro de $U$. lactuca conforme o design apresentado (12 tanques com densidade entre 2 e $10 \mathrm{~kg} \mathrm{~m}^{-3}$ de macroalga) seria capaz de filtrar, em menos de uma hora todo o nitrogênio dissolvido na água oriundo de $1 \mathrm{~kg}$ de ração para tilápias. Já para filtração de todo fósforo seriam necessárias aproximadamente 7 horas.

Figura 2: Representação da capacidade de filtração do biofiltro de macroalgas Ulva lactuca, mostrando o tempo de retenção da água no biofiltro para total retenção dos nutrientes dissolvidos.

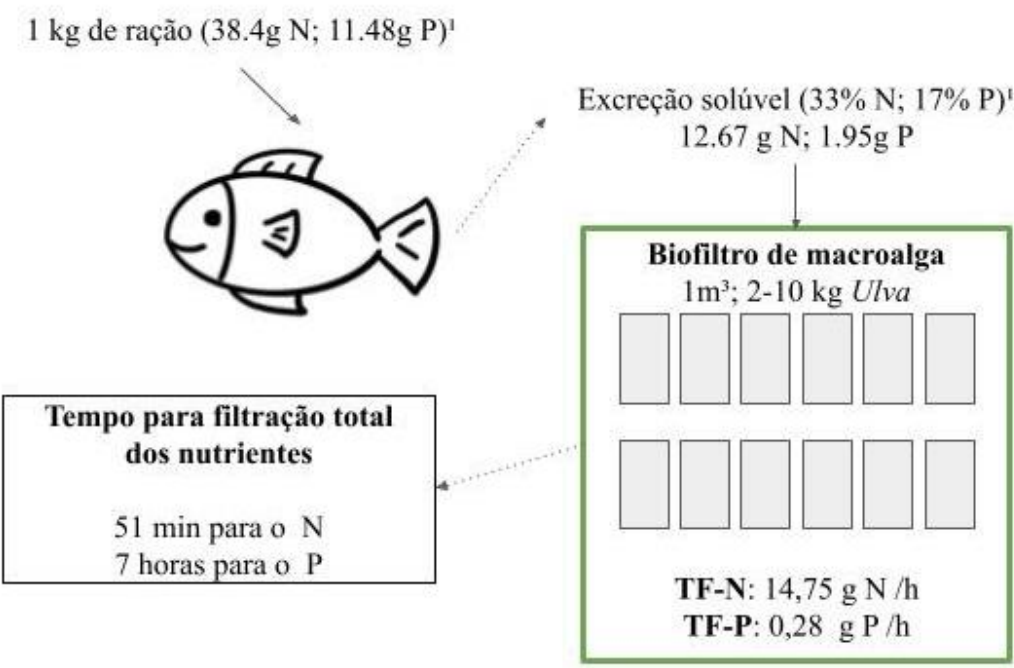

1 informações de Montanhini e Ostrensky (2015). Fonte: Autores (2022).

A elevada taxa de filtração dos nitrogenados pela macroalga evidencia a necessidade de balanceamento do sistema, tanto na oferta de nutrientes para a macroalga, como o ajuste nas outras etapas de filtração. O sucesso de sistemas de AMTI está relacionado à escolha e arranjo dos organismos no sistema de modo que o resíduo de um cultivo seja aproveitado por outros organismos sem ocorrer acúmulo ou falta de um determinado nutriente no sistema (Neori, et al., 2000; Soto, 2009; Chopin, 2010).

A Ulva lactuca demonstra uma elevada capacidade de absorção de nitrogênio inorgânico dissolvido, no entanto, a capacidade de filtração do fósforo foi 53 vezes menor. Neste sentido, para se evitar o acúmulo de fósforo e desequilibrar todo o sistema de AMTI, outros organismos de níveis tróficos diferentes devem ser incorporados à produção. A utilização de AMTI na produção do camarão Macrobrachium rosenbergii, com a incorporação de mais níveis tróficos ao sistema de produção,

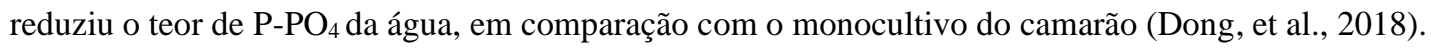

Nos tratamentos com menor densidade de estocagem de alga o índice de retirada de nutrientes foi maior. Este fato pode estar relacionado ao maior acesso à luz. $\mathrm{O}$ adensamento pode reduzir a disponibilidade de luz para a macroalga pois pode favorecer a sobreposição do material (Duke, et al., 1986; Neori, et al., 1991). A disponibilidade ideal de luz é um dos principais fatores para o crescimento da Ulva (Coutinho \& Zingmark, 1993; Rautenberger, et al., 2015).

O adensamento afeta o crescimento e a taxa fotossintética de Ulva (Jiang, et al., 2019), podendo levar a um acúmulo de pigmentos fotosintéticos, para compensar a menor disponibilidade luminosa (Revilla-Lovano, et al., 2021). A intensidade 
luminosa afetou a expressão de genes relacionados à síntese de carotenóides em Ulva prolifera (He, et al., 2020). De acordo com Cui et al. (2015), a intensidade luminosa ideal para o crescimento da Ulva pode variar de $70 \mathrm{a} 140 \mu \mathrm{mol} \cdot \mathrm{m}^{-2} \cdot \mathrm{s}^{-1}$. Neste sentido, a elevada irradiância registrada durante o período experimental possivelmente contribuiu para o desempenho das algas como biofiltro. De maneira, cultivos com alta relação superfície/volume favorecem a exposição dos talos à luz e facilitam a absorção de nutrientes, contribuindo assim para o seu crescimento.

A movimentação de água também é determinante para o crescimento de macroalgas (Hurd, 2000). A injeção de ar ou uso de bombas de circulação são artifícios que podem ser utilizados no design de biofiltros de macroalgas mais eficientes. A injeção de ar nos tanques de cultivo promove a estimulação mecânica, adição de $\mathrm{CO} 2$ e disponibiliza nutrientes com a movimentação da água (Traugott, et al., 2020).

O desempenho do biofiltro não foi alterado em função das densidades de estocagem testadas. Mesmo que o aumento da densidade, dentro dos limites estudados, prejudique a capacidade de absorção da macroalga e reduza o índice de filtração, o aumento da biomassa de organismos nas densidades maiores manteve a funcionalidade do biofiltro. Em sistemas de AMTI, onde o biofiltro além de realizar a remoção de nutrientes da água contribui para a produção de biomassa rentável para o sistema, é de grande importância a utilização de uma densidade de estocagem que proporcione uma alta produção de biomassa. Neste sentido, a escolha da densidade de estocagem ideal de um biofiltro em sistemas de AMTI deve ser balizada pela elevada absorção de nutrientes e alta produção de biomassa.

\section{Conclusão}

A variação da densidade de estocagem da Ulva lactuca entre 2 e $10 \mathrm{~kg} \cdot \mathrm{m}^{-3}$ não altera a eficiência de filtração e a taxa de filtração de nutrientes. No entanto, o aumento na densidade de estocagem prejudicou o índice de filtração de nitrogênio e fósforo, indicando que o maior adensamento reduz a capacidade de filtração de nutrientes da macroalga.

\section{Agradecimentos}

Os autores agradecem à empresa d'Alga Aquicultura Urbana por ceder as instalações e a estrutura do AMTI para a realização do experimento.

\section{Referências}

Adharini, R. I., Murwantoko, M., Probosunu, N., Setiawan, R. Y., \& Satriyo, T. B. (2021). The effectiveness of seaweeds as biofilter for reducing wastewater nutrient and preventing water pollution from hybrid grouper culture. Jurnal Ilmiah Perikanan dan Kelautan, 13(2). 10.20473/jipk.v13i2.28105

Al-Hafedh, Y. S., Alam, A., \& Buschmann, A. H. (2015). Bioremediation potential, growth and biomass yield of the green seaweed, Ulva lactuca in an integrated marine aquaculture system at the Red Sea coast of Saudi Arabia at different stocking densities and effluent flow rates. Reviews in Aquaculture, 7(3), 161-171. 10.1111/raq.12060

Ben-Ari, T., Neori, A., Ben-Ezra, D., Shauli, L., Odintsov, V., \& Shpigel, M. (2014). Management of Ulva lactuca as a biofilter of mariculture effluents in IMTA system. Aquaculture, 434, 493-498. 10.1016/j.aquaculture.2014.08.034

Boyd, C.E. Guidelines for aquaculture effluent management at the farm-level. (2003). Aquaculture, 226(1), 101-112. 10.1016/S0044-8486(03)00471-X

Boyd, C. E., \& Queiroz, J. F. (2004). Manejo das condições do sedimento do fundo e da qualidade da água e dos efluentes de viveiros. In: Cyrino, J. E. P., Urbinati, E. C., Fracalossi, D. M. et al. (Eds.) Tópicos especiais em piscicultura de água doce tropical intensiva. (pp. 25-43). Jaboticabal: Sociedade Brasileira de Aquacultura e Biologia Aquática,

Chopin T., Buschmann A. H., Halling C., Troell M., Kautsky N., \& Neori A. (2001) Integrating seaweeds into aquaculture systems: a key towards sustainability. Journal of Phycology, 37,75-986. 10.1046/j.1529-8817.2001.01137.x

Chopin, T. (2010). Integrated multi-trophic aquaculture. Advancing the aquaculture. Agenda: Workshop Proceedings. OECD Pblishing. 195-217. 10.1787/9789264088726-em 
Coutinho, R., \& Zingmark, R. (1993). Interactions of light and nitrogen on photosynthesis and growth of the marine macroalga Ulva curvata (Kützing) De Toni. Journal of experimental marine biology and ecology, 167(1), 11-19. 10.1016/0022-0981(93)90180-V

Cui, J., Zhang, J., Huo, Y., Zhou, L., Wu, Q., Chen, L., \& He, P. (2015). Adaptability of free-floating green tide algae in the Yellow Sea to variable temperature and light intensity. Marine pollution bulletin, 101(2), 660-666. 10.1016/j.marpolbul.2015.10.033

Dong, X., Lv, L., Zhao, W., Yu, Y., \& Liu, Q. (2018). Optimization of integrated multi-trophic aquaculture systems for the giant freshwater prawn Macrobrachium rosenbergii. Aquaculture Environment Interactions, 10, 547-556. 10.3354/aei00287

Duke, C.S., Lapointe, B.E., \& Ramus, J. (1986). Effects of light on growth, rubpcase activity and chemical composition of ulva species (chlorophyta) Journal of phycology, 22(3), 362-370. 10.1111/j.1529-8817.1986.tb00037.x

FAO (2020) The State of World Fisheries and Aquaculture 2020. Sustainability in action. Rome. 10.4060/ca9229en

Goddek, S., Delaide, B. P., Joyce, A., Wuertz, S., Jijakli, M. H., Gross, A., \& Keesman, K. J. (2018). Nutrient mineralization and organic matter reduction performance of RAS-based sludge in sequential UASB-EGSB reactors. Aquacultural engineering, 83, 10-19. 10.1016/j.aquaeng.2018.07.003

Hayashi, L., Yokoya, N.S., Ostini, S., Pereira, R.T., Braga, E. S., \& Oliveira, E. C. (2008). Nutrients removed by Kappaphycus alvarezii (Rhodophyta, Solieriaceae) in integrated cultivation with fishes in re-circulating water. Aquaculture, 277(3),185-191 10.1016/j.aquaculture.2008.02.024

He, Y., Ye, Y., \& Shen, S. (2020). Effects of light and salinity on carotenoid biosynthesis in Ulva prolifera. Acta Oceanologica Sinica, 39(10), 50-57. $10.1007 / \mathrm{s} 13131-020-1577-1$

HURD, C.L. (2000). Water motion, marine macroalgal physiology, and production. Journal of Phycology, 36(3), 453-472. 10.1046/j.1529-8817.2000.99139.x

Jiang, H., Gong, J., Lou, W., \& Zou, D. (2019). Photosynthetic behaviors in response to intertidal zone and algal mat density in Ulva lactuca (Chlorophyta) along the coast of Nan'ao Island, Shantou, China. Environmental Science and Pollution Research, 26(13), 13346-13353. 10.1007/s11356-019-04775-1

Montanhini Neto, R., \& Ostrensky, A. (2015). Nutrient load estimation in the waste of Nile tilapia Oreochromis niloticus (L.) reared in cages in tropical climate conditions. Aquaculture Research, 46(6), 1309-1322. 10.1111/are.12280

Msuya, F. E., \& Neori, A. (2008). Effect of water aeration and nutrient load level on biomass yield, N uptake and protein content of the seaweed Ulva lactuca cultured in seawater tanks. Journal of Applied Phycology, 20(6), 1021-1031 10.1007/s10811-007-9300-6

Neori, A., Shpigel, M., \& Ben-Ezra, D. (2000). A sustainable integrated system for culture of fish, seaweed and abalone. Aquaculture 186(3-4), 279-291. 10.1016/S0044-8486(99)00378-6

Neori, A., Cohen, I., \& Gordin, H. (1991) Ulva lactuca biofilters for marine fishpond effluents. II. Growth rate, yield and C: N ratio. Botanica Marina, 34(6), 483-490. 10.1515/botm.1991.34.6.483

Oliva Teles, A., Couto, A., Enes, P., \& Peres, H. (2020). Dietary protein requirements of fish-a meta-analysis. Reviews in Aquaculture.12(3), $1445-1477$. 10.1111/raq.12391

Queirós, A. S., Circuncisão, A. R., Pereira, E., Válega, M., Abreu, M. H., Silva, A., \& Cardoso, S. M. (2021). Valuable Nutrients from Ulva rigida: Modulation by Seasonal and Cultivation Factors. Applied Sciences, 11(13), 6137. 10.3390/app11136137

Rautenberger, R., Fernandez, P. A., Strittmatter, M., Heesch, S., Cornwall, C. E., Hurd, C. L., \& Roleda, M. Y. (2015). Saturating light and not increased carbon dioxide under ocean acidification drives photosynthesis and growth in Ulva rigida (Chlorophyta). Ecology and evolution, 5(4), 874-888. $10.1002 /$ ece 3.1382

Revilla-Lovano, S., Sandoval-Gil, J. M., Zertuche-González, J. A., Belando-Torrentes, M. D., Bernardeau-Esteller, J., Rangel-Mendoza, L. K., \& del Carmen Ávila-López, M. (2021). Physiological responses and productivity of the seaweed Ulva ohnoi (Chlorophyta) under changing cultivation conditions in pilot large land-based ponds. Algal Research, 56, 102316. 10.1016/j.algal.2021.102316

Shahar, B., \& Guttman, L. (2021). Integrated biofilters with Ulva and periphyton to improve nitrogen removal from mariculture effluent. Aquaculture, 532, 736011. 10.1016/j.aquaculture.2020.736011

Soto, D. (2009). Integrated mariculture: a global review (No. 529). Food and Agriculture Organization of the United Nations (FAO). Rome, 183p

Traugott, H., Zollmann, M., Cohen, H., Chemodanov, A., Liberzon, A., \& Golberg, A. (2020). Aeration and nitrogen modulated growth rate and chemical composition of green macroalgae Ulva sp. cultured in a photobioreactor. Algal Research, 47, 101808. 10.1016/j.algal.2020.101808 